\title{
Surgical Ventricular Restoration (SVR) - Hope for Heart Failure Patients or Is it a Dilemma ?
}

\author{
F Ahmed, M Sharifuzzaman, PK Chanda \\ Department of Cardiac Surgery, National Heart Foundation Hospital \& Research Institute, \\ Dhaka, Bangladesh.
}

(Cardiovasc. j. 2010; 2(2) : 118-120)

Congestive heart failure $(\mathrm{CHF})$ has reached epidemic proportions. It is estimated that $1 \%$ of the western world carries the diagnosis of CHF. Improvements in the medical and surgical treatment of acute coronary syndromes have led to an increasing number of patients surviving with their disease. ${ }^{1}$ These improvements, combined with other advances in health and technology, have now allowed the average US life expectancy to rise to 77 years. As a result, approximately 5 million Americans currently live with heart failure and an additional 400,000 patients are newly diagnosed each year. ${ }^{2,3}$ Exact epidemiological data from most developing nations are not available. However, given recent urbanization and improvements in life expectancy, the prevalence of hypertension and coronary artery disease, the two leading causes of heart failure, have also increased in the developing world. Accordingly, it can be expected that the prevalence of heart failure is also increasing in Bangladesh as in other developing nations. ${ }^{4}$

Despite tremendous advances in the medical management of $\mathrm{CHF}$ associated with the use of Angiotensin-converting enzyme inhibitors, âblockers, Aldosterone antagonists, as well as biventricular pacemakers and implantable defibrillators, the gold standard for the treatment of end-stage CHF remains cardiac transplantation. This therapy is associated with a 50\% 10 year survival and an optimal quality of life for the fortunate recipients. However, limited donor organ availability has rendered cardiac transplantation an epidemiological trivial therapy as it can only be offered to approximately 2,200 patients per year in USA. It is estimated however, that in the US alone, 60,000 patients could benefit from a new heart. Interestingly, $95 \%$ of the heart transplants worldwide are performed in North America and Europe, while less than 5\% occur in the three heavily populated continents, namely Asia, Africa and South America where over $75 \%$ of the world population resides.

In view of these limitations, several surgical alternatives for the treatment of $\mathrm{CHF}$ are being actively investigated. Some approaches involve an extension of current conventional cardiac operations like mitral valve repair while others seek to induce changes in the geometry of the left ventricle to render it a more efficient pump. ${ }^{5}$ Thus restoring myocardial perfusion (vessel), eliminating valvular regurgitation (valve), and restoring ventricular geometry (ventricle) has emerged as the first-line surgical approach (3V approach) to heart failure, because it can be performed in a single operation. ${ }^{6}$ Results of LV reconstruction have been favorable and consistent among groups, regardless of whether endoventricular circular patch plasty or a modified linear patch closure technique is used. ${ }^{7,8}$ Significant reductions in LV end-systolic volume index and improvements in ejection fraction, New York Heart Association class, and long-term survival have resulted. This surgical treatment, when combined with optimal medical management of heart failure, may now be as safe and effective as transplantation. Therefore, these modalities now form the first-line surgical therapy for heart failure, when applicable. ${ }^{9}$

The SVR procedure is by far the most extensively studied and applied technique for reshaping the dilated left ventricle. Its goal is to reduce left ventricular volume and create a more elliptical chamber by excluding scar in either akinetic or dyskinetic antero-apical and septal segments.

The validity of the SVR procedure was documented by the RESTORE (Reconstructive Endoventricular Surgery, returning Torsion Original Radius Elliptical Shape) group, an 11-center multinational 
group that evaluated the efficacy and durability of this procedure. In the most recent update from the group's registry, the outcome of 1198 postinfarction patients was reported. ${ }^{10}$ Concomitant procedures included bypass grafting in $95 \%$, mitral valve repair in $22 \%$ and mitral valve replacement in $1 \%$. Overall 30 -day mortality was $5.3 \%$ and need for perioperative mechanical support was uncommon $(<9 \%)$. Global systolic function improved with an increase in ejection fraction from $29 \pm 11 \%$ to $39 \pm 12 \%$ and a reduction in left ventricular end-systolic volume index from $80 \pm$ $51 \mathrm{ml} / \mathrm{m} 2$ to $57 \pm 34 \% \mathrm{ml} / \mathrm{m} 2$. Overall 5 year survival was $69 \pm 3 \%$. Predictors of death were ejection fraction d" $30 \%$, advanced NYHA class, age e" 75 years and left ventricular end-systolic volume indexe" $80 \mathrm{ml} / \mathrm{m}^{2}$. Remarkably, 5 year freedom from rehospitalization for $\mathrm{CHF}$ was $78 \%$ and $85 \%$ were in NYHA class I or II.

While the RESTORE's group data are compelling, randomized evaluation of this procedure is needed. To this end, the National Heart Lung and Blood Institute's multicenter, international, randomized STICH trial (Surgical Treatment for Ischemic Heart Failure) began enrolling patients with $\mathrm{CHF}$ and CAD in the spring of 2002. ${ }^{11}$ The goal is to determine whether a benefit over medical therapy can be found for coronary revascularization and whether this benefit can be enhanced by ventricular restoration surgery. One major focus of the STICH trial will be to determine the longterm outcome and durability of SVR.

The resulting trial included two major components. Patients in the Hypothesis 1 component were randomly assigned to receive either medical therapy alone or medical therapy plus CABG. The Hypothesis 1 component of the trial is ongoing. Patients in the Hypothesis 2 component were randomly assigned to receive medical therapy plus CABG or medical therapy plus CABG and surgical ventricular reconstruction.

STICH trial recently published in The New England Journal of Medicine (NEJM). ${ }^{12}$ Researchers reported results from "hypothesis two" in STICH, comparing surgical ventricular reconstruction (SVR) on top of CABG with CABG alone, show that the additional surgery successfully reduced the $L V$ volumes but had no impact on symptoms, exercise tolerance, deaths, or cardiac hospitalizations. "The findings of this study do not support the use of SVR in the population studied", Dr Robert H Jones (Duke University, Durham, $\mathrm{NC}$ ) and colleagues conclude in their paper.

STICH hypothesis two ended up enrolling 1000 patients with coronary disease suitable to CABG surgery, an ejection fraction of $35 \%$ or less, and dominant anterior LV dysfunction suitable for SVR. The SVR procedure, performed either on- or offpump, involved an anterior left ventriculotomy centered in the zone of anterior asynergy, a suture encircling the scar, then a cinching of the suture to bring the healthy portions of the ventricular walls in contact with one another. Following randomization and subsequent surgeries, patients were followed for a median of 48 months.

As Jones showed during a late-breaking clinicaltrials session, SVR in 501 patients successfully reduced end-systolic volume index to a greater degree than did CABG surgery in the remaining 499 patients ( $19 \%$ vs $6 \%$ ). But the surgical reshaping had no effect on improvement in angina and heartfailure symptoms or on six-minute walk test, all of which improved to a similar degree in both groups. Rates for the primary outcome of death from any cause combined with cardiac hospitalizations were also almost identical between the CABG and CABG / SVR groups, at 59\% and 58\%, respectively. Fatal events and cardiac hospitalizations were again mirrored between the two groups.

The hallmark of technical competence after a surgical procedure requires reaching goals that comply with yardsticks of procedural success. For SVR, favorable clinical results followed approximately $40 \%$ volume reduction below control levels in more than 1500 cases in 12 worldwide centers. In contrast, STICH reduced ventricular volume 19\%in 161 patients, and 96 centers were required to achieve this end point. No volume studies occurred in $66 \%$ of patients and an invalid echo-based monitoring method was used in the others. These limitations did not prevent STICH end point interpretation that SVR should be abandoned because CABG achieved similar results. The "Evidence-based Medicine" analysis used to justify this conclusion does not consider how results relate to evaluation of non-approved volume outcomes in only $33 \%$ of patients or how experience influences end point validity. 
We believe that differences between STICH outcomes and worldwide SVR data displaying greater volume reduction indicate that the wrong operation, using the wrong volume measurement monitors, was done on the wrong patients and resulted in the wrong conclusions. This outcome deprives cardiologists of understanding the potential role of volume reduction to treat $\mathrm{CHF}$ from ischemic dilated cardiomyopathy and demonstrates that the goals of evidence based medicine were not achieved. This analysis of STICH report flaws and conclusions suggests the trial should retract the NEJM report or reanalyze data to achieve meaningful results. The target goal must (1) exclude all patients with invalid echocardiographic volume measurements, (2) include only patients in whom regional nonviability of greater than $35 \%$ akinesia is documented by nuclear medicine scans, (3) quantify all patients with greater than $30 \%$ volume reduction by CMR (cardiac magnetic resonance) study, and (4) report only patients with "acceptable" volume reduction by CMR at 4 months. The STICH trial must address each of these questions because misguided STICH conclusions contradict the role of augmented ventricular volume as the surrogate for the natural history of increasing morbidity and mortality in dilated hearts. Without this action, the STICH trial conclusions simply show that statisticians can defy nature from a flawed database. ${ }^{13}$

Speculating on the possible reasons for the failure of SVR, despite numerous studies pointing to benefits with this approach, the authors hypothesize that experienced surgeons may have randomized only patients in whom SVR seemed less necessary, choosing to ensure that patients who they truly believed might benefit could get SVR directly, rather than risk randomization to the non-SVR groups.

\section{References:}

1. Szucs, TD. The growing healthcare burden of CHF. $J$ Renin Angiotensin Aldosterone Syst 2000; 1(Suppl 1):2-6.
2. http://www.nhlbi.nih.gov/health/dci/Diseases/HF/Hf. WhatIs.html.

3. Miller LW, Missov ED. Epidemiology of heart failure. Cardiol Clin 2001; 19:547-55.

4. Ali Ahmad, Kabir Zaman, A.K.M. Monowarul Islam, Abdul Momen. DEFEEAT: Practical Tips for the management of chronic heart failure. Bangladesh Heart Journal 2008; 23 (1): 48-55.

5. Goldstein DL, Smego D, Michler RE. Surgical aspects of congestive heart failure. Heart Fail Rev 2006; 11:17192.

6. Athanasuleas CL, Buckberg GD, Stanley AW, Siler W, Dor V, Di Donato M, et al. Surgical ventricular restoration in the treatment of congestive heart failure due to post-infarction ventricular dilation. J Am Coll Cardiol 2004; 44:1439-45.

7. Dor V, Sabatier M, Di Donato M, Maioli M, Toso A, Montiglio F. Late hemodynamic results after left ventricular patch repair associated with coronary grafting in patients with postinfarction akinetic or dyskinetic aneurysm of the left ventricle. J Thorac Cardiovasc Surg 1995; 110:1291-301.

8. Menicanti L, Di Donato M. The Dor procedure: what has changed after fifteen years of clinical practice? $J$ Thorac Cardiovasc Surg 2002; 124:886-90.

9. Spoor MT, Bolling SF. Nontransplant surgical options for heart failure. In: Cohn LH, Edmunds LH Jr, eds. Cardiac Surgery in the Adult. New York: McGrawHill, 2003:1639-48.

10. Athanasuleas CL, Stanley AWH Jr, Buckberg GD, Dor V, Di Donato M, Blackstone EH. Surgical anterior ventricular endocardial restoration in the dilated remodeled ventricle after anterior MI. RESTORE group. J Am Coll Cardiol 2001; 37:1199-209.

11. Doenst T,Velazquez EJ, Beyersdorf F,Michler R, Menicanti L ,Di Donato M,et al. To STICH or not to STICH: We know the answer, but do we understand the question? J Thorac Cardiovasc Surg 2005; 129:246-49.

12. Jones RH, Velazquez EJ, Michler RE, Sopko G, Oh JK, O'Connor CM, et al. Coronary bypass surgery with or without surgical ventricular reconstruction. $N$ Engl $J$ Med 2009; 360:1705-17.

13. Gerald D. Buckberg and Constantine L. Athanasuleas. The STICH trial: Misguided conclusions. J Thorac Cardiovasc Surg 2009; 138: 1060-64. 